\title{
Image-Quality Prediction of Synthetic Aperture Sonar Imagery
}

\author{
David P. Williams \\ NATO Undersea Research Centre \\ Viale San Bartolomeo 400, 19126 La Spezia (SP), Italy \\ williamsenato.nurc.int
}

\begin{abstract}
This work exploits several machine-learning techniques to address the problem of image-quality prediction of synthetic aperture sonar (SAS) imagery. The objective is to predict the correlation of sonar ping-returns as a function of range from the sonar by using measurements of sonar-platform motion and estimates of environmental characteristics. The environmental characteristics are estimated by effectively performing unsupervised seabed segmentation, which entails extracting wavelet-based features, performing spectral clustering, and learning a variational Bayesian Gaussian mixture model. The motion measurements and environmental features are then used to learn a Gaussian process regression model so that ping correlations can be predicted. To handle issues related to the large size of the data set considered, sparse methods and an out-of-sample extension for spectral clustering are also exploited. The approach is demonstrated on an enormous data set of real SAS images collected in the Baltic Sea.
\end{abstract}

Key words: Image-Quality Prediction, Synthetic Aperture Sonar (SAS), Gaussian Process Regression, Spectral Clustering, Sparse Methods, Out-of-Sample Extension, Variational Bayesian Gaussian Mixture Models, Unsupervised Seabed Segmentation, Large Data Sets.

\section{Introduction}

This work is an application paper. It exploits several machine learning techniques to address the problem of synthetic aperture sonar image-quality prediction.

Synthetic aperture sonar (SAS) provides high-resolution imaging of underwater environments by coherently summing sonar ping-returns. The correlation between successive pings at a given range (i.e., distance from the sonar) provides a measure of the success of the SAS processing, as this quantity is directly proportional to the signal-tonoise ratio [1].

Processing sonar returns into SAS imagery is a computationally intensive procedure. The ability to do this processing in near real-time onboard a sonar-equipped autonomous underwater vehicle (AUV) is a considerable challenge. Therefore, when tasked to survey a large area of seabed, an AUV will follow a pre-programmed route, with the SAS image-processing done post-mission. The route specified for the AUV assumes that a sufficiently high level of image quality will be attainable up to a certain range. 
However, the ability to successfully reconstruct SAS images — and in turn, the achievable area coverage - depends on the motion of the sonar-equipped platform and on the environmental characteristics of the area (such as the seabed-type and the water properties). Excessive platform motion will limit the range for which the coherent summation of pings is possible. Similarly, if the seabed is soft (e.g., muddy), less sonar energy will return to the receivers, also limiting the range to which a SAS image can be reconstructed successfully.

The objective of this work is to predict the SAS image-quality — in terms of the correlation of sonar pings - as a function of range from the sonar. The goal is to make this prediction - without performing the computationally-intensive SAS processing - by using measurements of platform motion and estimates of environmental characteristics. If the range to which the resulting SAS imagery will be of sufficient quality can be predicted, the route of an AUV can be adapted in-mission to both maximize its coverage rate and prevent missing areas of coverage. Moreover, the study can be used to understand the conditions for which SAS processing fails.

To achieve the stated goal, environmental characteristics are estimated by effectively performing unsupervised seabed segmentation, which entails extracting wavelet-based features [2], performing spectral clustering [3,4], and learning a variational Bayesian Gaussian mixture model [5]. Motion measurements collected onboard the platform and environmental features are then used to learn a Gaussian process regression model [6] so that predictions of the ping correlation can be made. To handle issues related to the large size of the data set considered, sparse methods [7] and an out-of-sample extension [8] for spectral clustering are also exploited. The approach is demonstrated on an enormous measured data set of real SAS images spanning a total area of approximately $44 \mathrm{~km}^{2}$ in the Baltic Sea.

The remainder of the paper is organized in the following manner. Sec. 2 briefly reviews the aspects of SAS processing relevant for the problem under study. Sec. 3 describes the process by which environmental features are extracted, which relies on spectral clustering and a variational Bayesian Gaussian mixture model. In Sec. 4, Gaussian process regression for the SAS image-quality prediction task is discussed. Sec. 5 shows experimental results obtained on a data set of real SAS images. Concluding remarks and directions for future work are given in Sec. 6.

\section{Synthetic Aperture Sonar (SAS)}

The high-resolution imaging of underwater environments provided by synthetic aperture sonar (SAS) can be used for applications such as mine detection, seabed classification, and the laying of gas or oil pipelines.

A SAS system transmits a broad-band signal such that each location on the seafloor is insonified by multiple pings. The ping returns are recorded onboard the sonar-equipped platform, such as an autonomous underwater vehicle (AUV). In order to reconstruct a SAS image, the returns are coherently summed by accounting for the time delay of signals at different ranges from the platform. The correlation between successive pings (which is directly proportional to the signal-to-noise ratio [1]) at a given range can be computed, with this quantity providing a measure of the success of the SAS processing. 
The displaced phase-center antenna (DPCA) method [1] is a popular data-driven approach used to reconstruct SAS imagery. The algorithm reconstructs a SAS image from $N_{i}^{p}$ collected pings by block-processing in the range direction at $N_{i}^{r}$ adaptivelydetermined DPCA range centers and windows. (For the data set considered in this work, the mean number of DPCA ranges per image was 12.2416.) A byproduct of the processing is the correlation of each pair of consecutive sonar pings at each DPCA range.

For the $i$-th SAS image, there will be $N_{i}^{p}$ correlation values at each of the $N_{i}^{r}$ DPCA ranges. To obtain a more robust summary measure of the correlation, we compute the mean correlation value (over the $N_{i}^{p}$ correlation values) at each of the $N_{i}^{r}$ ranges. It is these $N_{i}^{r}$ mean correlation values of the $i$-th SAS image that we wish to predict in this work.

\subsection{Features for Predicting Ping Correlation}

The ability to successfully perform SAS processing depends not only on the motion of the sonar platform (e.g., AUV), but also on the environmental characteristics of the seabed and the properties of the water through which the signals propagate [9].

In this work, platform motion measurements and seabed-type estimates are used as features to predict the ping correlation as a function of range. Platform motion is recorded onboard the vehicle via an inertial navigation system (INS), and hence readily accessible. The $d_{m}=5$ motion measurements used in this study are the roll, pitch, and yaw of the vehicle (i.e., rotations of the vehicle about three orthogonal axes), and the speed of the vehicle in the longitudinal and transverse directions.

The environmental features used in this study are based on an unsupervised seabed segmentation algorithm, and are related to the proportion of seabed area that belongs to each of $k$ different seabed-types at each range. The manner in which these features are extracted is described in detail in Sec. 3.

\section{Extraction of Environmental Features}

In this work, the "atomic" unit for seabed segmentation is assumed to be a $2 \mathrm{~m} \times 2 \mathrm{~m}$ area of seabed. That is, each $2 \mathrm{~m} \times 2 \mathrm{~m}$ area of seabed corresponds to one data point. This particular size was chosen as a compromise among several factors. The larger the area chosen, the more likely that a single data point will have the unfavorable property of containing multiple types of seabed. However, if the area is too small, the distinguishing characteristics of the seabed that indicate a certain seabed-type may be lost.

The proposed unsupervised seabed segmentation algorithm consists of three main steps. First, a vector of features based on a wavelet decomposition are extracted for each $2 \mathrm{~m} \times 2 \mathrm{~m}$ area of seabed. Spectral clustering is then applied, which transforms the data into a new, lower-dimensional space via an eigendecomposition. Lastly, a variational Bayesian Gaussian mixture model is learned using the transformed data. Seabed segmentation is effected in this step by assigning each data point to the mixture component that maximizes its posterior probability. Environmental features are then extracted from the result of the seabed segmentation. 


\subsection{Wavelet Features}

The proposed set of wavelet-based features consists of $d_{w}=16$ features that are derived from the coefficients of a biorthogonal wavelet decomposition [2] of each $2 \mathrm{~m} \times 2 \mathrm{~m}$ SAS image block (i.e., data point).

A wavelet decomposition of an image results in a set of decomposition coefficients that are computed via a cascade of filtering and subsampling. Each wavelet coefficient corresponds to a unique orientation and scale pair. The coefficients associated with one such pair comprise a "sub-image." In this work, we employ a compactly-supported biorthogonal spline wavelet and perform a five-scale decomposition, which results in 16 such sub-images. The features that are used for the seabed segmentation correspond to the quadratic mean (i.e., root-mean-square (RMS) value) of the wavelet coefficient amplitudes of each sub-image.

This particular set of features was chosen because it can successfully capture the distinguishing textural properties of the seabed. Namely, the wavelet-coefficient energy will be large when the orientation and scale match the orientation and scale of highenergy texture components in an image block (i.e., data point) [2].

\subsection{Spectral Clustering}

Overview Spectral clustering $[3,4]$ is a clustering method that uses an eigendecomposition to obtain a lower-dimensional embedding of a set of training data points. If additional data points were added to the data set, the eigenvectors would need to be recomputed anew. However, an out-of-sample extension for new data points in the context of spectral clustering has been proposed in [8] that avoids re-calculating the eigenvectors.

The eigendecomposition in question is of a matrix composed of distances between data points. Therefore, when spectral clustering is to be applied to large data sets, computational and memory issues can arise. A common way to ameliorate these issues is to construct the relevant matrix to be sparse and to subsequently employ a sparse eigensolver [7].

In this work, modifications to the standard spectral clustering approach are taken to address certain aspects encountered in the task at hand. Among these enhancements are the "sparsification" of key matrices to handle very large data sets [7], an automatic self-tuning of a parameter in the requisite affinity matrix [10], an out-of-sample extension to embed data points not present when the eigendecomposition is performed [8], and a decision to distinguish between the number of eigenvectors retained and the number of clusters desired. We integrate these various extensions into the standard spectral clustering algorithm of [4] in the presentation that follows.

Algorithm Let $\boldsymbol{w}_{i} \in \mathbb{R}^{d_{w}}$ denote a vector of $d_{w}$ features representing the $i$-th data point. For the following, consider a data set of $N_{b}^{t r}$ data points, $\mathcal{D}^{t r}=\left\{\boldsymbol{w}_{i}\right\}_{i=1}^{N_{b}^{t r}}$, that we wish to cluster into $k$ clusters. ${ }^{1}$ With the foresight that we will be considering outof-sample data points later, we define quantities as follows.

\footnotetext{
${ }^{1}$ At this stage in the overall application, a data point corresponds to a $2 \mathrm{~m} \times 2 \mathrm{~m}$ SAS image block of seabed, $N_{b}^{t r}=271,250$, and $d_{w}=16$.
} 
Define the distance between a pair of data points to be

$$
D\left(\boldsymbol{w}_{i}, \boldsymbol{w}_{j}\right)=D_{i j}=\left(\boldsymbol{w}_{i}-\boldsymbol{w}_{j}\right)^{T}\left(\boldsymbol{w}_{i}-\boldsymbol{w}_{j}\right),
$$

and define

$$
\widetilde{D}\left(\boldsymbol{w}_{i}, \boldsymbol{w}_{j}\right)=\widetilde{D}_{i j}= \begin{cases}D\left(\boldsymbol{w}_{i}, \boldsymbol{w}_{j}\right), & \text { if } \boldsymbol{w}_{i} \in T_{j} \text { or } \boldsymbol{w}_{j} \in T_{i} ; \\ 0, & \text { otherwise, }\end{cases}
$$

where $T_{i}$ is the set of $t$ nearest data points in $\mathcal{D}^{t r}$ to $\boldsymbol{w}_{i}$ (i.e., the $t$ nearest neighbors). By defining $\widetilde{D}_{i j}$ in this way, we ensure that a matrix constructed from these elements will be sparse and symmetric.

Define the affinity that expresses the similarity between a pair of data points to be

$$
A\left(\boldsymbol{w}_{i}, \boldsymbol{w}_{j}\right)=A_{i j}= \begin{cases}\exp \left\{-\widetilde{D}\left(\boldsymbol{w}_{i}, \boldsymbol{w}_{j}\right) / \sigma_{i} \sigma_{j}\right\}, & \text { if } \widetilde{D}\left(\boldsymbol{w}_{i}, \boldsymbol{w}_{j}\right) \neq 0 \\ 0, & \text { otherwise }\end{cases}
$$

where the self-tuning parameter $\sigma_{i}$ is defined as [10]

$$
\sigma_{i}=\frac{1}{t_{i}} \sum_{j=1}^{N_{b}^{t r}} \widetilde{D}\left(\boldsymbol{w}_{i}, \boldsymbol{w}_{j}\right)
$$

and $t_{i}$ is the number of non-zero elements of $\widetilde{D}\left(\boldsymbol{w}_{i}, \boldsymbol{w}_{j}\right)$ for $\boldsymbol{w}_{j} \in \mathcal{D}^{t r}$.

Next define

$$
B\left(\boldsymbol{w}_{i}\right)=B_{i}=\sum_{j=1}^{N_{b}^{t r}} A\left(\boldsymbol{w}_{i}, \boldsymbol{w}_{j}\right)
$$

to be the sum of the affinities associated with $\boldsymbol{w}_{i}$.

For the data set of $N_{b}^{t r}$ (in-sample) data points, $\mathcal{D}^{t r}$, the soon-to-be-defined matrices $\mathbf{D}, \widetilde{\mathbf{D}}, \mathbf{A}, \mathbf{B}$, and $\widetilde{\mathbf{A}}$ will each be of size $N_{b}^{t r} \times N_{b}^{t r}$.

Let the $i j$-th element of the matrix $\mathbf{D}$ be $D_{i j}$ from (1). Similarly, let the $i j$-th elements of the sparse and symmetric matrices $\widetilde{\mathbf{D}}$ and $\mathbf{A}$ be $\widetilde{D}_{i j}$ from (2) and $A_{i j}$ from (3), respectively. Define $\mathbf{B}$ to be the diagonal matrix whose $i$-th entry is $B_{i}$ from (5). Define the $i j$-th element of the normalized affinity matrix $\widetilde{\mathbf{A}}$ to be

$$
\widetilde{A}\left(\boldsymbol{w}_{i}, \boldsymbol{w}_{j}\right)=\widetilde{A}_{i j}=\frac{A_{i j}}{\sqrt{B_{i} B_{j}}}
$$

so that the matrix can be written $\widetilde{\mathbf{A}}=\mathbf{B}^{-1 / 2} \mathbf{A} \mathbf{B}^{-1 / 2}$.

Next find the $m$ eigenvectors $-\boldsymbol{e}_{1}, \boldsymbol{e}_{2}, \cdots, \boldsymbol{e}_{m}$ - of $\widetilde{\mathbf{A}}$ associated with the $m$ largest eigenvalues $-\lambda_{1}, \lambda_{2}, \cdots, \lambda_{m}-$ and stack them in columns to form the matrix $\mathbf{E}=\left[\boldsymbol{e}_{1} \boldsymbol{e}_{2} \cdots \boldsymbol{e}_{m}\right] \in \mathbb{R}^{N_{b}^{t r} \times m}$. Because $\widetilde{\mathbf{A}}$ is sparse, a sparse eigensolver can be used to perform the eigendecomposition.

Then construct the matrix $\mathbf{Z} \in \mathbb{R}^{N_{b}^{t r} \times m}$ to be a re-normalized version of $\mathbf{E}$ whose rows have unit length, so

$$
Z_{i j}=\frac{E_{i j}}{\sqrt{\sum_{j=1}^{m} E_{i j}^{2}}}
$$


Treat each row of $\mathbf{Z}$ as a data point in $\mathbb{R}^{m}$, meaning the original feature vector $\boldsymbol{w}_{i} \in \mathbb{R}^{d_{w}}$ has effectively been transformed into a new vector $\boldsymbol{z}_{i} \in \mathbb{R}^{m}$.

Now consider a new (i.e., out-of-sample) data point, $\boldsymbol{w}_{*} \in \mathbb{R}^{d_{w}}$, that was not in the original data set $\mathcal{D}^{t r}$. We adopt the out-of-sample extension of spectral clustering proposed in [8] to obtain the embedding of $\boldsymbol{w}_{*}$.

For $i=1,2, \ldots, N_{b}^{t r}$, compute $\widetilde{A}\left(\boldsymbol{w}_{*}, \boldsymbol{w}_{i}\right)$ using (6). The out-of-sample spectralclustering embedding (in one dimension) of $\boldsymbol{w}_{*}$ is given by [8]

$$
e_{* j}=\frac{1}{\lambda_{j}} \sum_{i=1}^{N_{b}^{t r}} E_{i j} \widetilde{A}\left(\boldsymbol{w}_{*}, \boldsymbol{w}_{i}\right)
$$

The complete spectral-clustering embedding of a new (out-of-sample) data point $\boldsymbol{w}_{*}$, when the top $m$ eigenvectors are retained, is then $\boldsymbol{z}_{*}=\left[z_{* 1} z_{* 2} \cdots z_{* m}\right]$, where unit-norm normalization is performed via $z_{* j}=e_{* j} / \sqrt{\sum_{j=1}^{m} e_{* j}^{2}}$.

The last step of spectral clustering is to cluster the normalized (in-sample) embedded data, $\left\{\boldsymbol{z}_{i}\right\}_{i=1}^{N_{b}^{t r}}$, into $k$ clusters via a clustering algorithm. The cluster assignment of the original data point $\boldsymbol{w}_{i}$ is exactly the cluster assignment of $\boldsymbol{z}_{i}$. In this work, a variational Bayesian Gaussian mixture model (GMM) is used as the clustering algorithm.

Discussion Spectral clustering is well-suited for the task of seabed segmentation because an area of seabed corresponding to a particular seabed-type (e.g., sand ripples) often undergoes a gradual change in appearance in SAS imagery. Spectral clustering will recognize that such a "chain" of data points should belong to the same cluster [11].

In the standard formulation of spectral clustering, rigorous theoretical underpinnings justify that the number of eigenvectors retained, $m$, is set equal to the number of clusters, $k$ to be found $[4,11]$. However, we choose to go against this convention for our particular application. As noted in [10], when dealing with noisy data, the "ideal" block-diagonal affinity matrix is not attained. As a result, using the eigen-gap [4] to determine the number of clusters in the data is unreliable, because the progression of the eigenvalues may not exhibit a distinct jump in magnitudes.

For our application, the number of clusters (i.e., seabed types) is not known a priori. Therefore, rather than adopting a questionable approach with spectral clustering to determine the number of clusters, we transfer this burden onto the GMM. This decision makes sense for several reasons.

By doing so, we can choose to retain a very small number of eigenvectors (e.g., $m=2$ ), which accelerates the eigendecomposition computation. Choosing the number of clusters based on the eigen-gap would instead require a complete eigendecomposition (or at least many more eigenvectors to be computed), which is computationallyintensive for the very large data set considered in this work.

By embedding the data into such a low-dimensional space via spectral clustering, the complexity (i.e., number of parameters to estimate) of the subsequent GMM is also greatly reduced, which simplifies learning. In addition, the variational Bayesian GMM approach adopted in this work naturally determines the number of mixture components in a principled way. Moreover, the algorithm can ascertain this number in a single run, which is again valuable with such a large data set. 


\subsection{Variational Bayesian Gaussian Mixture Model}

It is well-known that a Gaussian mixture model (GMM) can accurately model an arbitrary continuous distribution. Variational Bayesian methods [12] can be used to learn full distributions of a GMM's parameters (i.e., the mixing proportions, means, and covariances). Such an approach can also determine the appropriate number of mixture components represented by the data by selecting the model (i.e., number of mixture components) associated with the highest evidence [12].

However, when the number of data points is large and the number of features is small - the scenario in our application - this approach will result in an excessively large number of mixture components. Moreover, the time-consuming model estimation must be conducted numerous times (for each possible number of mixture components).

In contrast, an alternative variational Bayesian approach referred to as component splitting [5] can, in a single run, learn a GMM and determine the appropriate number of mixture components that are represented in the data. Therefore, this method is employed in this work to learn a GMM, from a set of $N_{b}^{t r} m$-dimensional data points. ${ }^{2}$

The variational Bayesian component splitting approach [5] for learning a GMM begins with a single mixture component. A splitting test is applied to the component to determine if there is sufficient evidence that two components exist within the present one. If there is, the component is split into two, increasing the number of mixtures in the model. The method then recursively performs the splitting test on each new component until no new components are formed. At each iteration, variational Bayesian updateequations are applied to the parameters of only the component being split. Because of space constraints, we do not provide more specific details of the method here.

Upon learning the GMM, each data point is assigned to the mixture component (i.e., "cluster") for which its posterior probability is maximum. For the task at hand, each mixture component can be viewed as a unique seabed-type. Therefore, this clusterassignment step effects a segmentation of the seabed into different seabed-types.

\subsection{Environmental Features from Seabed Segmentation}

Let the number of $2 \mathrm{~m} \times 2 \mathrm{~m}$ seabed blocks in the $i$-th SAS image that are within the $j$-th DPCA range window be $N_{b}^{(i, j)}$. Suppose each seabed block has been assigned to a component of the $k$-component GMM. Let $N_{b}^{(i, j, k)}$ be the number of seabed blocks within the $j$-th DPCA range window of the $i$-th SAS image that was assigned to the $k$-th GMM component. The $k$-th environmental feature for the $j$-th DPCA range window of the $i$-th SAS image is then defined to be the fraction

$$
\boldsymbol{x}_{(i, j)}^{e}(k)=\frac{N_{b}^{(i, j, k)}}{N_{b}^{(i, j)}} .
$$

The entire environmental feature extraction process for an example image is shown in Fig. 1.

\footnotetext{
${ }^{2}$ At this stage in the overall application, a data point corresponds to a $2 \mathrm{~m} \times 2 \mathrm{~m}$ SAS image block of seabed, $N_{b}^{t r}=271,250$, and $m=2$.
} 


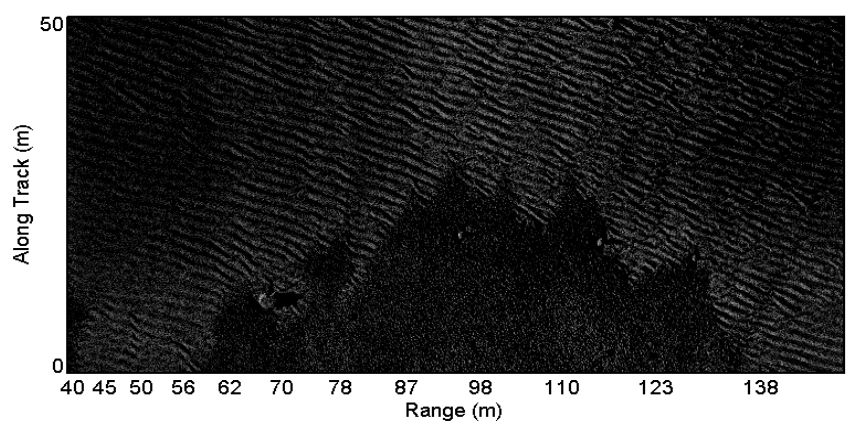

(a) SAS image

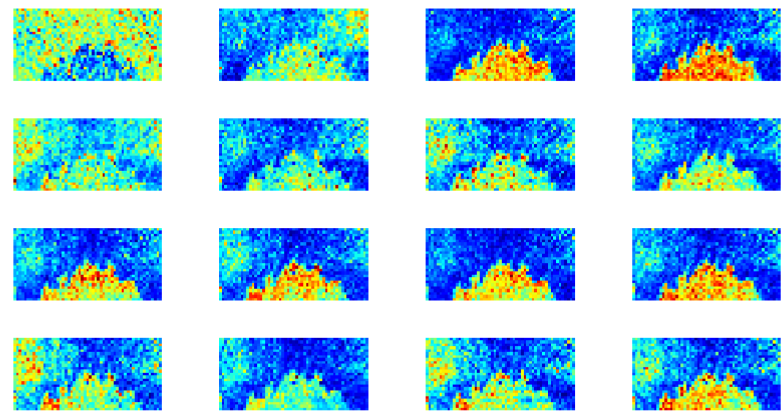

(b) Wavelet features
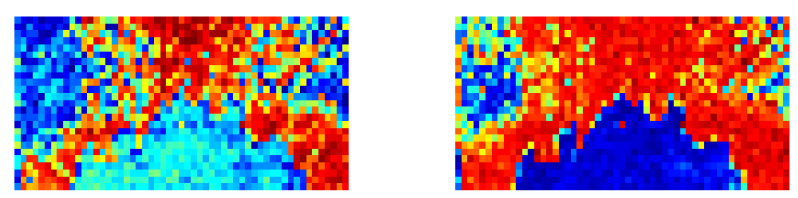

(c) Eigenvectors

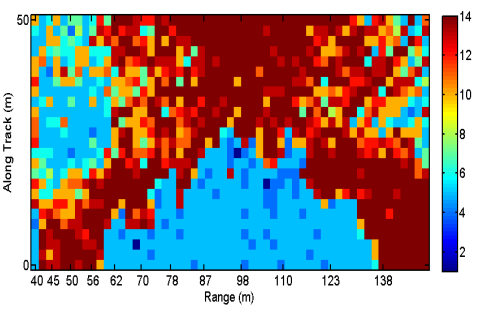

(d) Segmentation

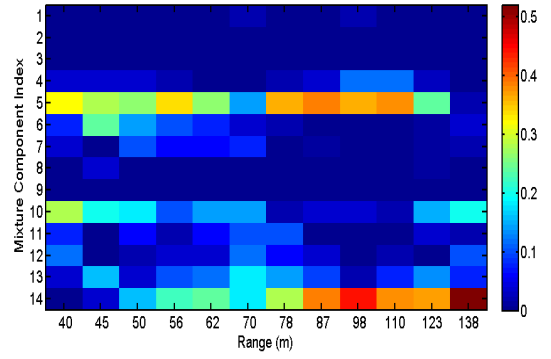

(e) Environmental features

Fig. 1. Example of environmental feature extraction process for a test image. From a SAS image (a), 16 wavelet features (b) are extracted for each $2 \mathrm{~m} \times 2 \mathrm{~m}$ area of seabed. An out-of-sample extension for spectral clustering is applied, reducing the 16 wavelet features to 2 eigenvector features (c). A previously-learned variational Bayesian GMM is used to assign the eigen-data to mixture components, effecting a segmentation (d) of the seabed. The proportions of data points within each range window of interest that are assigned to each mixture component are subsequently used as environmental features (e) in a GP regression model. 


\section{Gaussian Process Regression}

Let $\boldsymbol{x}_{i} \in \mathbb{R}^{d}$ denote an input (column) vector of $d$ features representing the $i$-th data point, and $y_{i}$ its corresponding noisy scalar output. For the following, consider a data set of $N_{r}^{t r}$ data points, $\left\{\boldsymbol{x}_{i}, y_{i}\right\}_{i=1}^{N_{r}^{t r}}=\{\mathbf{X}, \boldsymbol{y}\}$, that we wish to learn a regression model for that describes the relationship between an input $\boldsymbol{x}$ and its (noisy) output $y=f(\boldsymbol{x})+\epsilon$, where $\epsilon$ is assumed to be independent identically distributed Gaussian noise with variance $\sigma_{n}^{2}{ }^{3}$ With a regression model learned, one can make predictions for an unknown function $f_{*}=f\left(\boldsymbol{x}_{*}\right)$, or noisy output $y_{*}$, given any input $\boldsymbol{x}_{*}$. To complete this task, we employ a Gaussian process (GP) regression model [6], which provides an elegant, fully Bayesian solution to the problem.

A GP is a collection of random variables, any finite number of which has a joint Gaussian distribution. A GP is fully specified by a mean function and a covariance function. The mean function is typically taken to be the zero function, which we also assume. The (kernel) covariance function $K_{i j}$, which expresses the covariance between the values of the noisy outputs $y_{i}$ and $y_{j}$, is usually chosen to be a Gaussian covariance function of the form

$$
K_{i j}=k\left(y_{i}, y_{j}\right)=\sigma_{s}^{2} \exp \left\{-\frac{1}{2}\left(\boldsymbol{x}_{i}-\boldsymbol{x}_{j}\right)^{T} \boldsymbol{\Phi}^{-1}\left(\boldsymbol{x}_{i}-\boldsymbol{x}_{j}\right)\right\}+\sigma_{n}^{2} \delta_{i j}
$$

where $\delta_{i j}$ is a Kronecker delta function, $\sigma_{s}^{2}$ and $\sigma_{n}^{2}$ are the signal variance and noise variance, respectively, and $\boldsymbol{\Phi}$ is a diagonal matrix of length-scale hyperparameters, $\phi_{1}, \ldots, \phi_{d}$.

The kernel function essentially provides a measure of similarity between pairs of data points; the length-scale hyperparameters weight the relative importance and contribution of each feature in measuring this similarity. Collecting these similarity measures for each pair of training data points then forms the kernel covariance matrix, $\mathbf{K}$, of the GP.

The kernel covariance function $K_{i j}$ drives the entire Gaussian process. In fact, the problem of learning a GP regression model is simply the problem of learning the kernel function hyperparameters $\Theta=\left\{\phi_{1}, \ldots, \phi_{d}, \sigma_{s}^{2}, \sigma_{n}^{2}\right\}$. The hyperparameters can be learned by maximizing the marginal likelihood (i.e., the evidence) [6],

$$
\log p(\boldsymbol{y} \mid \mathbf{X})=-\frac{1}{2} \boldsymbol{y}^{T} \mathbf{K}^{-1} \boldsymbol{y}-\frac{1}{2} \log |\mathbf{K}|-\frac{N_{r}^{t r}}{2} \log (2 \pi) .
$$

With $K_{i j}$ defined and $\Theta$ specified, making predictions with the GP regression model can be done analytically. Let the input vectors of new test data points for which predictions are desired be collected in the matrix $\mathbf{X}_{*}$. Under the Gaussian process prior (and the Gaussian noise model), the joint distribution of the observed (noisy) outputs $\boldsymbol{y}$ and the unknown (noisy) outputs of the new test points, $\boldsymbol{y}_{*}$, can be written

$$
p\left(\left[\begin{array}{c}
\boldsymbol{y} \\
\boldsymbol{y}_{*}
\end{array}\right] \mid \mathbf{X}, \boldsymbol{\Theta}, \mathbf{X}_{*}\right)=\mathcal{N}\left(\left[\begin{array}{c}
\boldsymbol{y} \\
\boldsymbol{y}_{*}
\end{array}\right] ;\left[\begin{array}{l}
\mathbf{0} \\
\mathbf{0}
\end{array}\right],\left[\begin{array}{cc}
\mathbf{K} & \mathbf{K}_{*} \\
\mathbf{K}_{*}^{T} & \mathbf{K}_{* *}
\end{array}\right]\right),
$$

\footnotetext{
${ }^{3}$ At this stage in the overall application, a data point corresponds to a particular range in a particular SAS image, and $N_{r}^{t r}=2,437$.
} 
where $\mathbf{K}_{*}$ is a matrix of covariances between the noisy outputs of the test data points and the observed outputs of the $N_{r}^{t r}$ data points, and $\mathbf{K}_{* *}$ is a matrix of covariances between the noisy outputs of the test data points. Conditioning (12) on the observed output values will result in another Gaussian distribution, namely

$$
p\left(\boldsymbol{y}_{*} \mid \boldsymbol{y}, \mathbf{X}, \boldsymbol{\Theta}, \mathbf{X}_{*}\right)=\mathcal{N}\left(\boldsymbol{y}_{*} ; \mathbf{K}_{*}^{T} \mathbf{K}^{-1} \boldsymbol{y}, \mathbf{K}_{* *}-\mathbf{K}_{*}^{T} \mathbf{K}^{-1} \mathbf{K}_{*}\right) .
$$

The key predictive equation for GP regression is given by (13). That is, the predictive means for the noisy outputs $\boldsymbol{y}_{*}$ at the new test points are $\overline{\boldsymbol{y}}_{*}=\mathbf{K}_{*}^{T} \mathbf{K}^{-1} \boldsymbol{y}$, while the predictive covariances are $\operatorname{cov}\left(\boldsymbol{y}_{*}\right)=\mathbf{K}_{* *}-\mathbf{K}_{*}^{T} \mathbf{K}^{-1} \mathbf{K}_{*}$, the diagonal elements of which conveniently provide error bars on the predictions.

\subsection{GP Regression for Predicting Ping Correlation}

In the application addressed in this work, GP regression is employed to predict the ping correlation value, $y$, as a function of range and other input features, $\boldsymbol{x}$. We consider three different GP models, each of which differs in the features that are employed. In all three models, the target output is the mean correlation value of each "data point" (i.e., image-range pair).

The data point corresponding to the $i$-th image's $j$-th DPCA range is represented in the three GPR models by

$$
\begin{aligned}
& \text { Model M: } \quad \boldsymbol{x}_{(i, j)}=\left[x_{(i, j)}^{r} \boldsymbol{x}_{(i, j)}^{m}\right] \\
& \text { Model E: } \quad \boldsymbol{x}_{(i, j)}=\left[x_{(i, j)}^{r} \boldsymbol{x}_{(i, j)}^{e}\right] \\
& \text { Model M+E: } \quad \boldsymbol{x}_{(i, j)}=\left[x_{(i, j)}^{r} \boldsymbol{x}_{(i, j)}^{m} \boldsymbol{x}_{(i, j)}^{e}\right] \text {, }
\end{aligned}
$$

where $x_{(i, j)}^{r}, x_{(i, j)}^{m}$, and $x_{(i, j)}^{e}$ are the scalar range, vector of $d_{m}$ motion features, and vector of $k$ environmental features, respectively, of the $i$-th image's $j$-th DPCA range.

It should be noted that the GP prediction can be made at any range. However, because we possess true measurements of the correlation at specific ranges for each image, we choose to make GP predictions only at those same locations so that the error can be computed.

\section{Experimental Results}

\subsection{Data Set}

In April-May 2008, the NATO Undersea Research Centre (NURC) conducted the Colossus II sea trial in the Baltic Sea off the coast of Latvia. During this trial, high-resolution sonar data was collected by the MUSCLE autonomous underwater vehicle (AUV). This AUV is equipped with a $300 \mathrm{kHz}$ sonar with a $60 \mathrm{kHz}$ bandwidth that can achieve an along-track image resolution of approximately $3 \mathrm{~cm}$ and an across-track image resolution of approximately $2.5 \mathrm{~cm}$. The sonar data was subsequently processed into synthetic aperture sonar (SAS) imagery. 
The data set consists of 8,097 SAS images. Each image covers $50 \mathrm{~m}$ in the alongtrack direction. The vast majority of images cover $110 \mathrm{~m}$ in the across-track (i.e., range) direction, typically from $40 \mathrm{~m}$ to $150 \mathrm{~m}$ away from the AUV. A small number of the images cover ranges as close as $20 \mathrm{~m}$ or as far away as $200 \mathrm{~m}$. The total area spanned by the entire data set of images is approximately $44 \mathrm{~km}^{2}$.

In this work, the $N_{s}=8,097 \mathrm{SAS}$ images contain a total of $N_{b}=10,984,025$ $2 \mathrm{~m} \times 2 \mathrm{~m}$ seabed blocks. There are $N_{r}=99,120$ image-range pairs for which we possess the (mean) ping correlation value (i.e., the quantity we wish to predict using motion and environmental features).

\subsection{Training Stage}

For the experiments conducted here, $N_{s}^{t r}=200$ of the images were randomly selected to be used as training images, with the remaining $N_{s}^{t s}=7,897$ images treated as testing images. Among the training images, there were a total of $N_{b}^{t r}=271,250$ seabed blocks and $N_{r}^{t r}=2,437$ image-range pairs.

The training procedure proceeded as follows. As described in Sec. 3.1, $d_{w}=16$ wavelet features were extracted for each of the $N_{b}^{t r} 2 \mathrm{~m} \times 2 \mathrm{~m}$ seabed blocks.

Spectral clustering as described in Sec. 3.2 was then performed on the $N_{b}^{t r}$ seabed blocks. Because of the huge number of "data points" considered, sparse methods were employed. Specifically, the matrix in (2) was made sparse such that only the $t=20$ nearest neighbors for each data point were retained; all other entries were set to zero. The top $m=2$ eigenvectors were retained from the eigendecomposition, meaning the data was effectively transformed from $\mathbb{R}^{d_{w}}$ to $\mathbb{R}^{m}$.

Next, the variational Bayesian component splitting method described in Sec. 3.3 was used to learn a GMM using the $N_{b}^{t r}$ data points in $\mathbb{R}^{m}$. The resulting GMM had $k=14$ mixture components. Each of the $N_{b}^{t r}$ data points was then assigned to the mixture component for which its posterior probability was a maximum. This step effected a segmentation of the seabeds of the training images.

The $k=14$ environmental features were then calculated for each of the $N_{r}^{t r}=2,437$ image-range pairs as described in Sec. 3.4.

Then, the three GP regression models defined in Sec. 4.1 were learned using the $N_{r}^{t r}=2,437$ "data points." The difference among the models was which set of features was used.

\subsection{Testing Stage}

For the experiments conducted here, the remaining $N_{s}^{t s}=7,897$ images were used as testing images. Among these testing images, there were a total of $N_{b}^{t s}=10,712,775$ seabed blocks and $N_{r}^{t s}=96,683$ image-range pairs.

For each of the $N_{b}^{t s}$ seabed blocks, wavelet features were extracted, the out-ofsample extension for spectral clustering described in Sec. 3.2 was applied, and assignment to the GMM component for which its posterior probability was a maximum was made; this last step effected a segmentation of the seabeds of the testing images. The $k=14$ environmental features were then calculated for each of the $N_{r}^{t s}$ image-range 
pairs. Finally, the correlation value for each of the $N_{r}^{t s}$ image-range pairs was predicted using each of the three learned GP regression models.

\subsection{Results}

In all results reported here, the term "error" refers to the absolute error of the ping correlation values, $\epsilon=\left|y^{\text {true }}-y^{\text {predicted }}\right|=\left|y_{*}-\bar{y}_{*}\right|$.

To compare the three GP regression models, the proportion of the $N_{r}^{t s}=96,683$ image-range pairs (i.e., "data points") for which each model obtained a lower error than the other models was computed. Because a set of image-range pairs are associated with each image, one can also compute the mean error (averaged across the image-range pairs belonging to an image) for each of the $N_{s}^{t s}=7,897$ testing images. This method of assessment is more realistic than comparing individual image-range pairs because in practice, one wishes to make predictions for all ranges of an image jointly, not just at one particular range. Prediction results in terms of the proportion of image-range pairs and also the proportion of images for which each model obtained a lower error than the other models are presented in Table 1.

Table 1. Proportion of image-range pairs (left portion of table) and proportion of images (right portion of table) for which each model obtained a lower prediction error than each competing model.

\begin{tabular}{|c|c|c|c|c|c|c|}
\hline \multirow[b]{3}{*}{ MODEL } & \multicolumn{3}{|c|}{ (IMAGE-RANGE PAIRS) } & \multicolumn{3}{|c|}{ (IMAGES) } \\
\hline & \multicolumn{3}{|c|}{\begin{tabular}{|l|} 
COMPETING MODEL \\
\end{tabular}} & \multicolumn{3}{|c|}{ COMPETING MODEL } \\
\hline & M & $\mathrm{E}$ & $\mathrm{M}+\mathrm{E}$ & M & $\mathrm{E}$ & $M+E$ \\
\hline Motion (M) & -—- & 0.4268 & 0.4015 & -—— & 0.3586 & 0.3109 \\
\hline ENVIRONMENT (E) & 0.5732 & - - & 0.4860 & 0.6414 & - - & 0.4541 \\
\hline MOTION \& ENVIRONMENT $(\mathrm{M}+\mathrm{E})$ & 0.5985 & 0.5140 & -—- & 0.6891 & 0.5459 & - \\
\hline
\end{tabular}

A main motivation for conducting this study was to predict the maximum range to which a SAS image could be successfully reconstructed to a sufficiently high level of quality. Therefore, the performance of the three GP regression models as a function of range is shown in Fig. 2. (Each range bin spans $10 \mathrm{~m}$, with the exception of the last bin, which runs from $150 \mathrm{~m}$ to $200 \mathrm{~m}$ since there were considerably fewer data points in that range of ranges.) The mean error (averaged across the image-range pairs that fell within each given range bin) is shown for each of the three GP regression models.

As can be seen from Table 1 and Fig. 2, the models that included the environmental features consistently perform the best. Of particular interest to our study, however, is prediction performance at long ranges. In this regime, the model incorporating both motion features and environmental features was most accurate.

The results presented above are for a very large data set and are only mean results. To more closely examine performance at an individual image level, we show the GP regression correlation predictions for three example SAS images, along with the actual SAS images, in Figs. 3-5.

The SAS image in Fig. 3(a) is notable because the near-range contains relatively flat seabed, while the far-range contains seabed structure (such as rocks and ridges). 


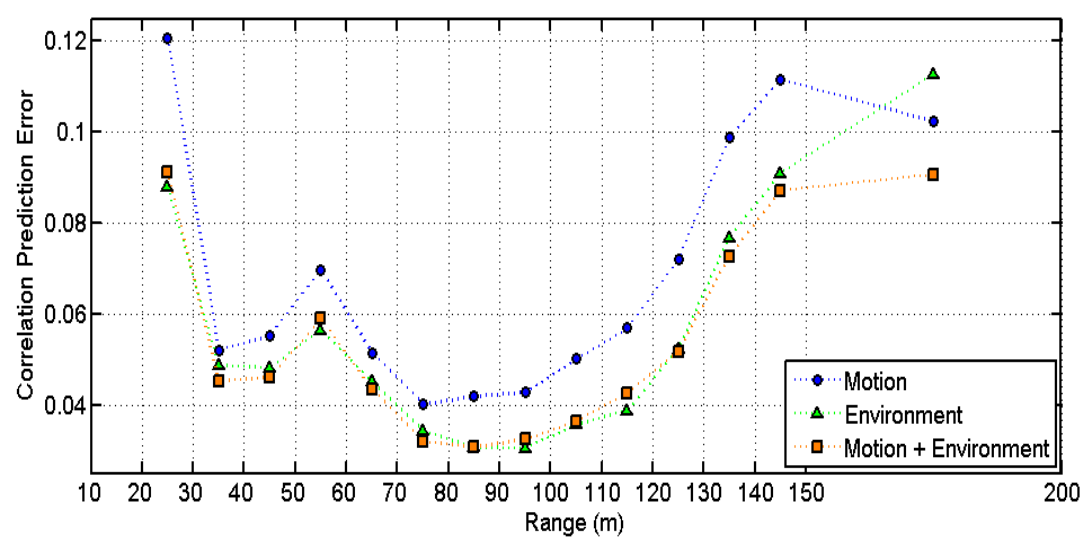

Fig. 2. Mean prediction error as a function of range bin for each GP regression model.

The presence of seabed structure counteracts the natural correlation degradation that typically occurs at longer ranges, thereby permitting high correlation values from being obtained. The GP regression models that account for environmental features are able to exploit this information and accurately predict the relatively high correlation values at the longer ranges.

\section{Conclusion}

This work was an application paper that exploited several machine-learning techniques to address the problem of synthetic aperture sonar (SAS) image-quality prediction. The problem is important because if the range to which the resulting SAS imagery will be of sufficient quality can be predicted, the route of an AUV can be adapted to both maximize its coverage rate and prevent missing areas of coverage. Moreover, the study can be used to better understand the conditions for which SAS processing fails.

To achieve the objective, environmental characteristics were estimated by effectively performing unsupervised seabed segmentation, which entailed extracting waveletbased features, performing spectral clustering, and learning a variational Bayesian Gaussian mixture model. Motion measurements (collected onboard the sonar platform) and environmental features were then used to learn a Gaussian process regression model so that predictions of the ping correlations could be made. To handle issues related to the large size of the data set, sparse methods and an out-of-sample extension for spectral clustering were also exploited. The approach was demonstrated on an enormous measured data set of real SAS images spanning a total area of approximately $44 \mathrm{~km}^{2}$.

The experimental results suggest that prediction of the SAS image-quality in terms of the correlation values via motion measurements and environmental characteristics is indeed feasible. The results also demonstrated that using both motion features and environmental features together achieved the best prediction performance at the long ranges of particular interest. Future work will seek to incorporate other environmental mea- 


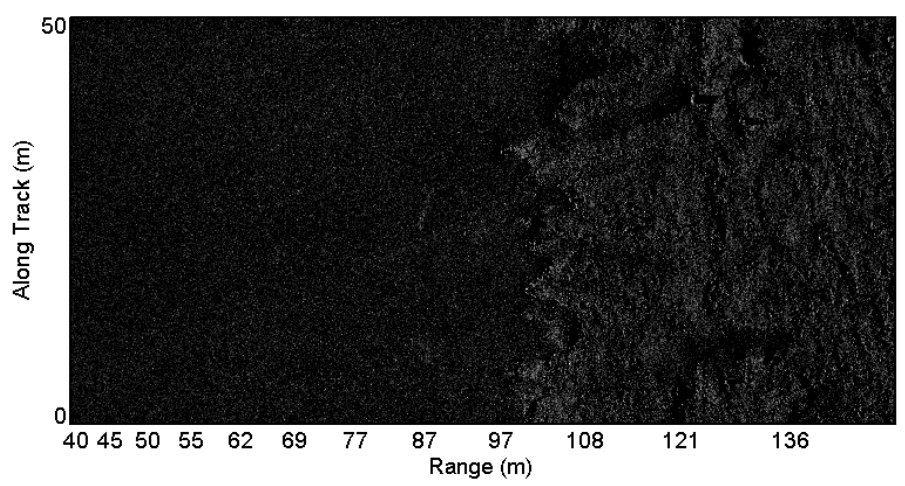

(a)

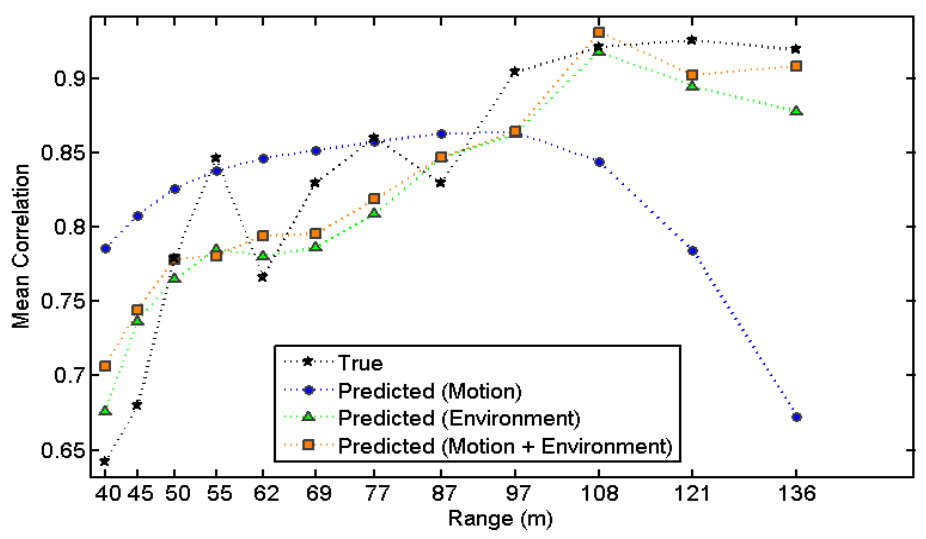

(b)

Fig. 3. A SAS image and its associated GP regression correlation predictions.

surements (e.g., wind speed, water temperature) as additional features in the regression model.

Often, before collecting any sonar data, one will possess rough a priori knowledge of the seabed-types over the area of interest, in the form of a seabed map. However, for the data set considered in this work, such a seabed map was not available. Therefore, to assess the feasibility of the image-quality prediction task, environmental features were extracted from the seabed segmentation that was performed on the data. Admittedly, this approach should produce the optimal performance, since the " $a$ priori" seabed knowledge is essentially assumed to be perfect. Future work will examine correlation-prediction performance when the environmental features are based on only a priori seabed knowledge possessed before data collection commences (rather than on the segmentation of the actual imagery that would not be available). 


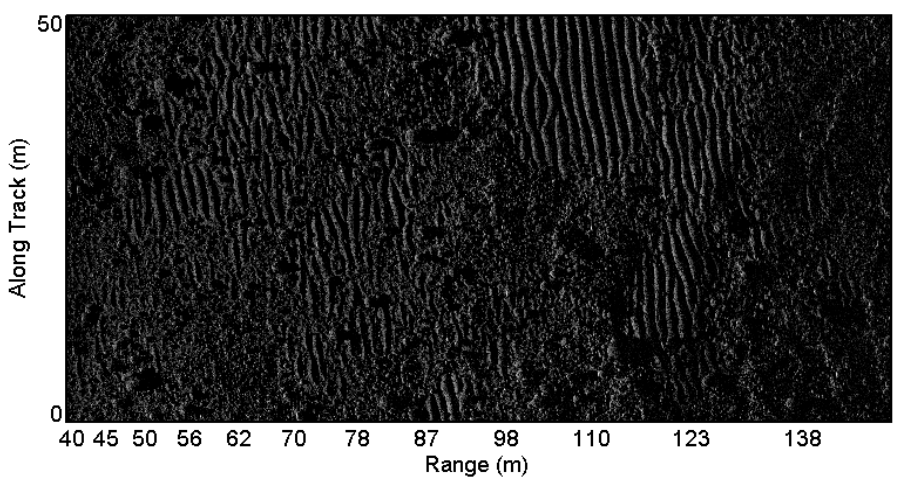

(a)

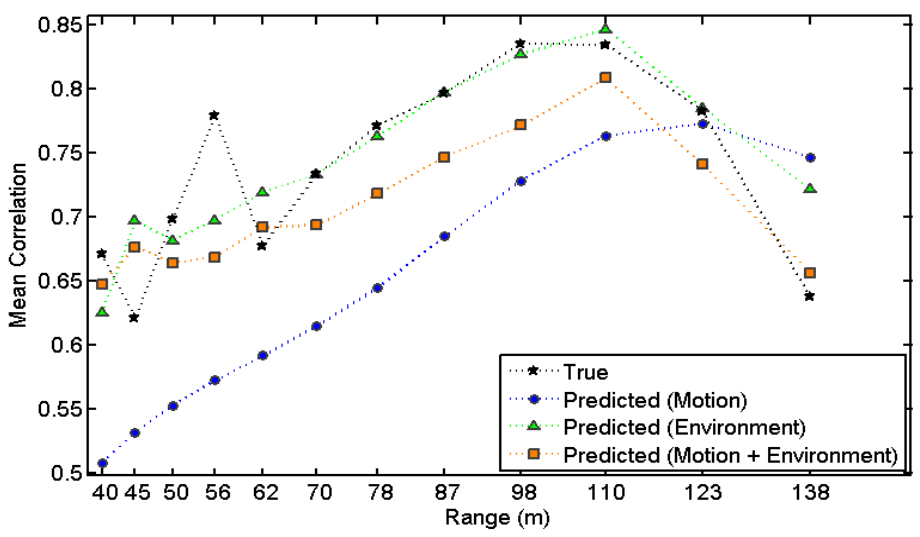

(b)

Fig. 4. A SAS image and its associated GP regression correlation predictions.

\section{References}

1. A. Bellettini and M. Pinto, "Theoretical accuracy of synthetic aperture sonar micronavigation using a displaced phase-center antenna," IEEE Journal of Oceanic Engineering, vol. 27, no. 4, pp. 780-789, 2002.

2. S. Mallat, A Wavelet Tour of Signal Processing. Academic Press, 1999.

3. M. Meilă and J. Shi, "Learning segmentation by random walks," in Advances in Neural Information Processing Systems. MIT Press, 2000, pp. 873-879.

4. A. Ng, M. Jordan, and Y. Weiss, "On spectral clustering: Analysis and an algorithm," in Advances in Neural Information Processing Systems. MIT Press, 2001, pp. 849-856.

5. C. Constantinopoulos and A. Likas, "Unsupervised learning of Gaussian mixtures based on variational component splitting," IEEE Transactions on Neural Networks, vol. 18, no. 3, pp. 745-755, 2007.

6. C. Rasmussen and C. Williams, Gaussian Processes for Machine Learning. MIT Press, 2006. 


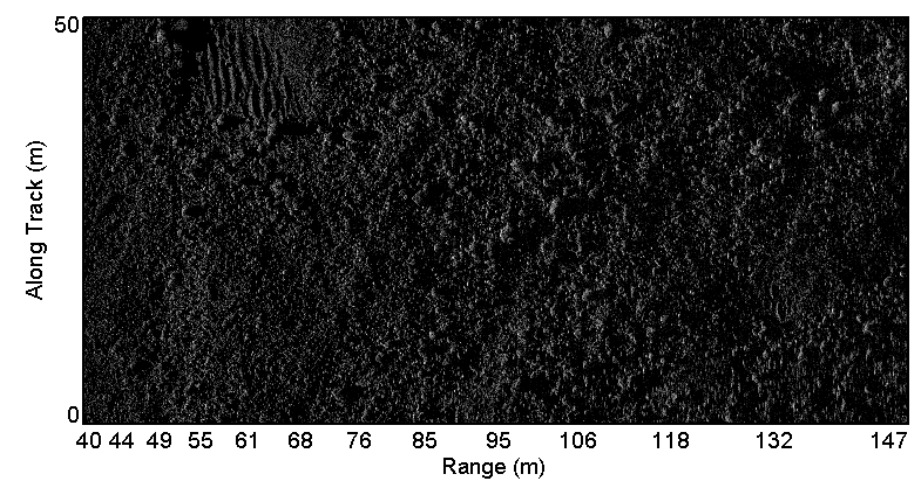

(a)

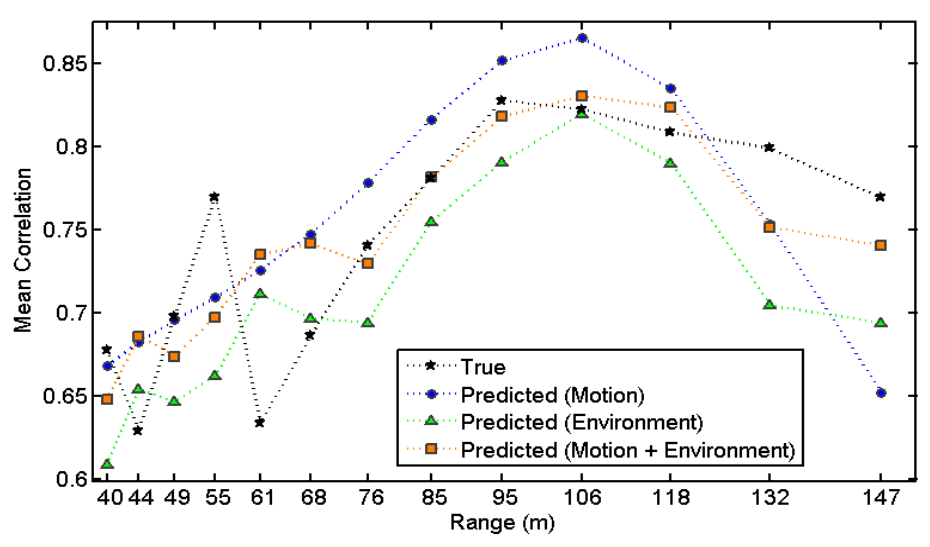

(b)

Fig. 5. A SAS image and its associated GP regression correlation predictions.

7. Y. Song, W. Chen, H. Bai, C. Lin, and E. Chang, "Parallel spectral clustering," in Proceedings of the European Conference on Machine Learning and Principles and Practice of Knowledge Discovery in Databases (ECML/PKDD), 2008.

8. Y. Bengio, J. Paiement, P. Vincent, O. Delalleau, N. Le Roux, and M. Ouimet, "Out-ofsample extensions for LLE, isomap, MDS, eigenmaps, and spectral clustering," in Advances in Neural Information Processing Systems. MIT Press, 2004, pp. 177-184.

9. M. Hayes and P. Gough, "Broad-band synthetic aperture sonar," IEEE Journal of Oceanic Engineering, vol. 17, no. 1, pp. 80-94, 1992.

10. L. Zelnik-Manor and P. Perona, "Self-tuning spectral clustering," in Advances in Neural Information Processing Systems. MIT Press, 2004, pp. 1601-1608.

11. U. von Luxburg, "A tutorial on spectral clustering," Statistics and Computing, vol. 17, no. 4, pp. 395-416, 2007.

12. M. Beal and Z. Ghahramani, "The variational Bayesian EM algorithm for incomplete data: Application to scoring graphical model structures,” Bayesian Statistics, vol. 7, pp. 453-464, 2003. 